\title{
Early and Successful Reconstruction of a Traumatic Bilateral Patellar Tendon Rupture: A Case Report
}

\author{
Mahmoud Jabalameli ${ }^{1}$, Majid Abedi ${ }^{1,}{ }^{,}$, Mehran $\operatorname{Radi}^{1}$, Hooman Yahyazadeh ${ }^{1}$ and Seyed Jalil Hamidi ${ }^{1}$ \\ ${ }^{1}$ Bone and Joint Reconstruction Research Center, Shafa Orthopedic Hospital, Iran University of Medical Sciences, Tehran, Iran \\ "Corresponding author: Bone and Joint Reconstruction Research Center, Shafa Orthopedic Hospital, Iran University of Medical Sciences, Tehran, Iran. Email: \\ majid.abedi844@gmail.com
}

Received 2018 August 12; Revised 2018 September 07; Accepted 2018 September 19.

\begin{abstract}
Traumatic bilateral patellar tendon rupture (BPTR) is a rare injury with low levels of clinical suspicion, especially in young adults. Since early diagnosis of the BPTR is the cornerstone of a successful management, BPTR reports are valuable as they raise awareness of the careful clinical and radiologic workout in similar cases. Here, we present a case of BPTR in a 34-year-old male following a motorcycle accident. At the emergency department, the necessary examinations including clinical, physical, and radiologic tests were performed and BPTR was diagnosed accordingly. The patient underwent reconstruction surgery the day after the injury. At the last follow-up, the patient was able to perform active straight leg raise with $130^{\circ}$ flexion and no extension lag. The superior results of BPTR reconstruction in this report further emphasize the importance of early diagnosis and repair of BPTR. Active straight leg raise test is necessary to perform for all patients with a history of an eccentric load of the knee extensor mechanism, and if positive, extensor mechanism rupture should be strongly suspected.
\end{abstract}

Keywords: Knee Extensor Mechanism, Bilateral Patellar Tendon Rupture, Trauma

\section{Introduction}

The patellar tendon is one of the components of the knee extensor mechanism that its rupture leads to the disruption of the knee extensor mechanism. Early diagnosis and surgical reconstruction of the patellar tendon tear is necessary to achieve early functional recovery (1).

Bilateral patellar tendon rupture (BPTR) is a rare injury and the majority of cases are reported in patients with predisposing tendon-weakening conditions such as systemic lupus erythematosus, rheumatoid arthritis, chronic renal failure, and corticosteroid use (2). Traumatic BPTR in young adults is even rarer and usually associates with low levels of clinical suspicion.

The diagnosis of BPTR could be easily made based on the clinical and radiologic examinations. BPTR patients usually present with pain, swelling, a palpable gap in the tendon, proximally placed patella, and loss or limitation of extension (3). However, the diagnosis could be challenging in specific conditions such as the presence of severe hematoma that frequently accompanies acute rupture of patellar tendon and may conceal the main diagnostic signs, including a palpable infrapatellar gap. For this reason, nearly $38 \%$ of the patellar tendon ruptures are misdiagnosed initially (4).

Since the early diagnosis of BPTR is the cornerstone of a successful management, raising awareness of the occurrence of this injury, even in young adults with no predisposing condition, is crucial as it ensures careful physical examinations in similar cases. Hence, we here report a case of BPTR following a motorcycle accident in a 34-year-old man with no predisposing condition.

\section{Case Presentation}

A 34-year-old healthy man was admitted to the emergency department of our hospital after a motorcycle accident. In the clinical examination, pain, swelling of both knees, and walking disability were noticed, with no history of tendon-weakening conditions such as previous trauma, systemic disorder, or steroid consumption. In the physical examination, straight leg raise testing demonstrated a bilateral impairment of terminal knee extension. Moreover, a palpable infrapatellar gap was observed in both patellae. No other injury was present and the patient was hemodynamically stable. Accordingly, the presumptive clinical 
diagnosis of BPTR was made. Plain radiographs demonstrated a proximal displacement of the patellae (Figure 1). Magnetic resonance imaging (MRI) showed a high-riding patella and defects in patellar tendons (Figure 2). The definitive diagnosis of BPTR was made based on the clinical and radiologic findings.

Subsequently, the surgical repair of the tendons was performed the day after the injury. At the surgery, the tendons were found ruptured through the inferior poles of the patella with disruption of the retinaculum thoroughly. The ruptures were repaired with Fiber-Wire suture in Krackow fashion to create a four-strand repair. These strands were passed through three longitudinal holes that were made near the articular surface of the patella. Then, in the knees hyperextension state, the sutures were stitched over the quadriceps tendon. The retinaculum tears were repaired using absorbable suture afterward. The repair stability was checked intra-operatively and $90^{\circ}$ passive flexion was obtained at the surgery.

Two cylinder casts were applied at the end of the operation in full knees extension for six weeks. The patient was advised to bear weight as tolerated and allow straight leg raise at three weeks. At the sixth week, the casts were removed and standard physiotherapy started for the patient. One year after the surgery, the patient was able to perform active straight leg raise with $130^{\circ}$ flexion and no extension lag.

\section{Discussion}

Patellar tendon rupture is a rare orthopedic injury. According to an epidemiological study of ligament and tendon lesions of the musculoskeletal system performed by Clayton and Court-Brown, the rate of patellar ligament rupture was $0.6 \%$ (5). Obviously, the rate of BPTR is even lower and generally associated with tendon-weakening comorbidities $(2,6)$. It is estimated that a force of 17.5 times the body weight is needed to tear a healthy patellar tendon (7) and only a few BPTR reports are available in healthy patients.

In 1978, Margles and Lewis reported a case of spontaneous BPTR without any apparently associated systemic disease (8). In 1979, Kamali reported the first traumatic BPTR in a 52-year-old man (9). In 1986, Webb and Toby reported a case of BPTR in an otherwise healthy 38-yearold male patient following a minor trauma. A flexion force against a contracted or contracting quadriceps muscle was proposed as the mechanism of injury (10). Subsequently, traumatic BPTR was reported in several studies al- though not frequently (11-15). Here, we introduced a new case of traumatic BPTR in a 38-year-old male that was diagnosed and managed successfully with early reconstruction surgery.

The reports of BPTR are important because they raise awareness of the devoting careful attention to its early diagnosis, as late diagnosis and repair usually result in an unfavorable outcome. The outcome of a patellar tendon rupture significantly depends on the interval between injury and repair. Early surgery is recommended for optimal results. This is best accomplished by accurate reapproximation of the tendon ends and repair of the torn extensor retinacula. An intensive rehabilitation program, emphasizing early range-of-motion exercises, progressive weight bearing, and quadriceps strengthening will improve the results of surgery. Delayed repair is associated with compromised results secondary to the loss of full knee flexion and reduced quadriceps strength although a functional extensor mechanism could still be reestablished (2).

Cree et al. (3) reported a case of missed BPTR in a 75year-old male following the slipping forward onto both knees. At the local emergency unit, no radiographs were obtained and he was diagnosed with a "soft tissue injury" and discharged with simple analgesics in spite of not being able to maintain a standing position even when physically lifted and helped. Since the patient was unable to straighten his leg voluntarily even 70 days after the initial injury, an orthopedic referral was made with a subsequent diagnosis of BPTR and the reconstruction was performed 81 days after the initial injury. Follow-up radiographs showed some continued patella alta. At the last follow-up, the patient had a bilateral extension lag of $5^{\circ}$, but retained the full range of motion (3). Similar inferior results of surgery were reported in other neglected cases of $\operatorname{BPTR}(15,16)$.

Although the diagnosis of BPTR is not usually challenging, due to the rarity of this injury, clinical suspicion is low and misdiagnosis is highly probable. As the delayed repair significantly affects the outcome of surgery, performing an active straight leg raise test is necessary to perform for all patients with a history of an eccentric load of the knee extensor mechanism. In case of a positive active straight leg raise test, the extensor mechanism of rupture should be strongly suspected and further evaluations should be performed. In challenging cases, such as patients not referred in the acute phase or the presence of severe hematoma, complementary imaging studies such as ultrasonography and MRI should be used to correctly rule out the possibility of the extensor mechanism of rupture. 

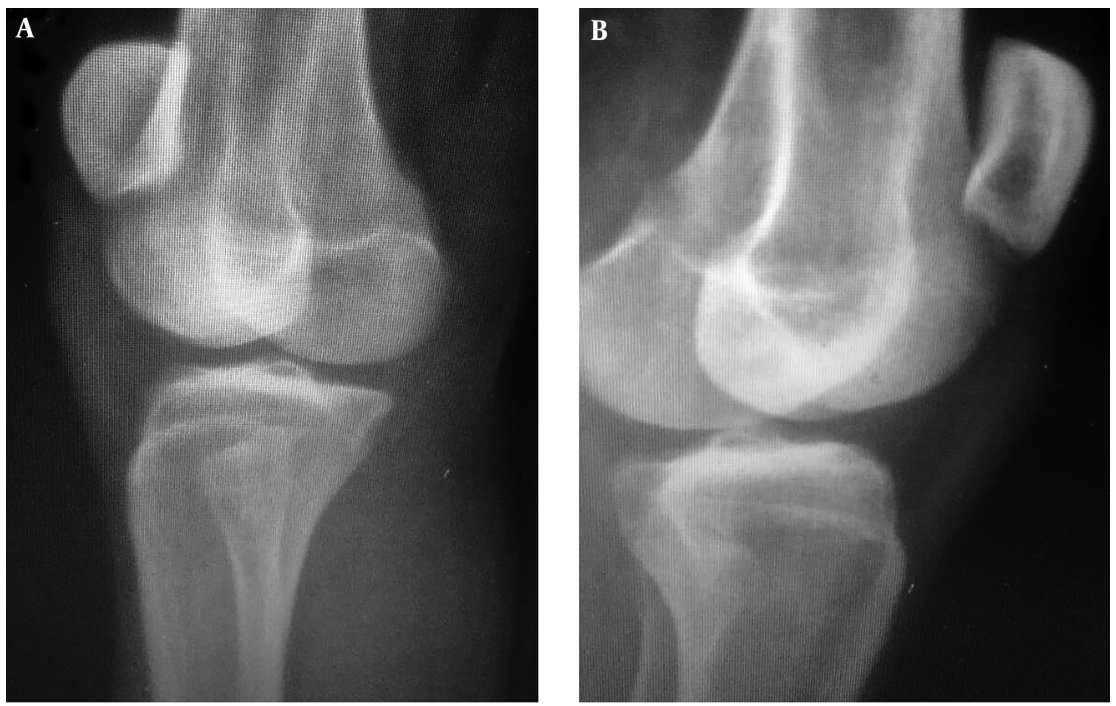

Figure 1. Preoperative lateral radiographs of (A) right and (B) left knee, showing proximal displacement of the patella.
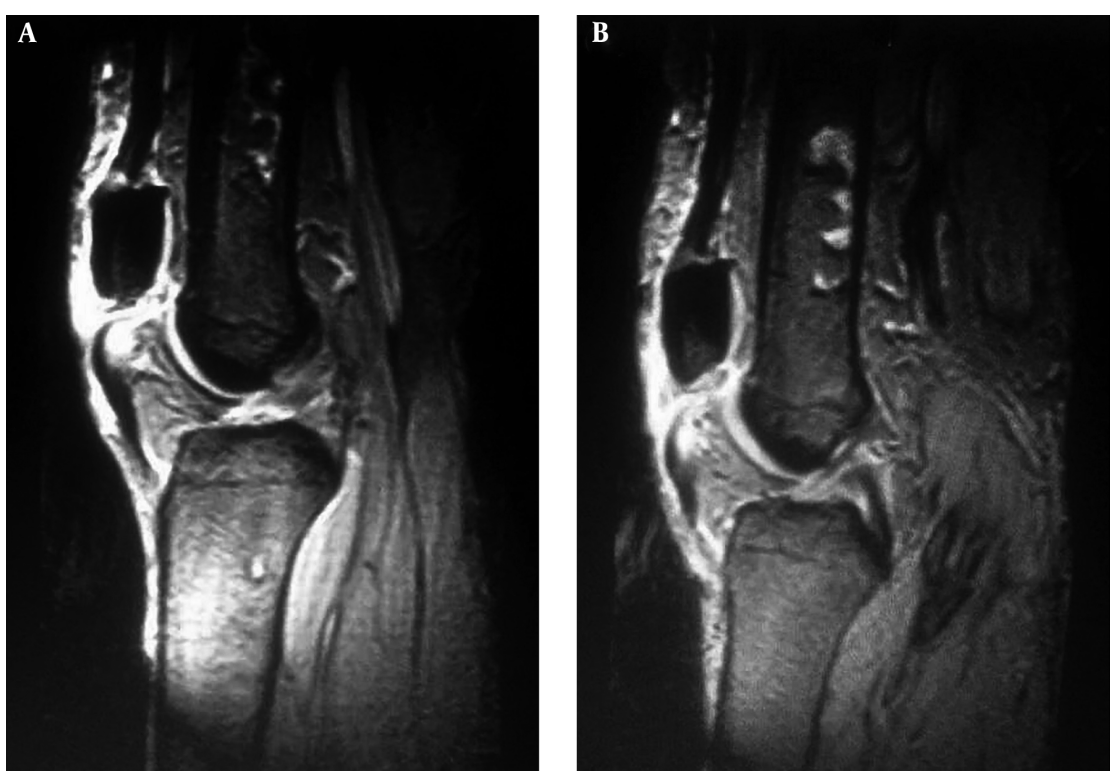

Figure 2. Magnetic resonance imaging (MRI) of (A) right and (B) left knee, showing defects in the inferior poles of the patellar tendons.

\section{References}

1. Duthon VB, Fritschy D. [Knee extensor mechanism ruptures]. Rev Med Suisse. 2011;7(304):1544-8. French. [PubMed: 21919393].

2. Matava MJ. Patellar tendon ruptures. J Am Acad Orthop Sur. 1996;4(6):287-96. doi: 10.5435/00124635-199611000-00001.

3. Cree C, Pillai A, Jones B, Blyth M. Bilateral patellar tendon ruptures: A missed diagnosis : Case report and literature review. Knee Surg Sports Traumatol Arthrosc. 2007;15(11):1350-4. doi:10.1007/s00167-007-0350-8. [PubMed: 17604980].
4. Siwek CW, Rao JP. Ruptures of the extensor mechanism of the knee joint. J Bone Joint Surg Am. 1981;63(6):932-7. [PubMed: 6985557].

5. Clayton RA, Court-Brown CM. The epidemiology of musculoskeletal tendinous and ligamentous injuries. Injury. 2008;39(12):1338-44. doi: 10.1016/j.injury.2008.06.021. [PubMed: 19036362].

6. Bagherifard A, Jabalameli M, Rezazadeh J, Ghaffari S, Tabrizian P. Simultaneous bilateral quadriceps tendon rupture following a low energy trauma in a male body builder with the history of anabolic - androgenic steroids consumption. Shafa Orthop J. 2018;5(2). doi: $10.5812 /$ soj.68635. 
7. Zernicke RF, Garhammer J, Jobe FW. Human patellar-tendon rupture. J Bone Joint Surg Am. 1977;59(2):179-83. [PubMed: 845201].

8. Margles SW, Lewis MM. Bilateral spontaneous concurrent rupture of the patellar tendon without apparent associated systemic disease: A case report. Clin Orthop Relat Res. 1978;(136):186-7. [PubMed: 729284].

9. Kamali M. Bilateral traumatic rupture of the infrapatellar tendon. Clin Orthop Relat Res. 1979;(142):131-4. [PubMed: 498625].

10. Webb LX, Toby EB. Bilateral rupture of the patella tendon in an otherwise healthy male patient following minor trauma. J Trauma. 1986;26(11):1045-8. [PubMed: 3783781].

11. Kellersmann R, Blattert TR, Weckbach A. Bilateral patellar tendon rupture without predisposing systemic disease or steroid use: A case report and review of the literature. Arch Orthop Trauma Surg. 2005;125(2):127-33. doi: 10.1007/s00402-004-0782-2. [PubMed: 15645271].

12. Ho HM, Lee WK. Traumatic bilateral concurrent patellar tendon rupture: An alterative fixation method. Knee Surg Sports Traumatol
Arthrosc. 2003;11(2):105-11. doi: 10.1007/s00167-002-0332-9. [PubMed: 12664203].

13. Tarazi N, O'Loughlin P, Amin A, Keogh P. A rare case of bilateral patellar tendon ruptures: A case report and literature review. Case Rep Orthop. 2016;2016:6912968. doi: 10.1155/2016/6912968. [PubMed: 27200200]. [PubMed Central: PMC4854987].

14. Hua-Ding L, Dao-Zhang C, Kun W, Chun Z, Shuang-Ming S. [Simultaneous bilateral patellar tendon rupture without predisposing systemic disease or steroid use: A case report]. Chinese JTraumatol. 2012;15:54-8. Chinese. doi: 10.3760/cma.j.issn.1008-1275.2012.01.011.

15. Koné S, Dogba E, Nguianbanda L, Kouassi A, Ledion A, Krah L, et al. Clinical case of bilateral rupture of the patellar tendon of the late diagnosis. Open J Orthopedics. 2017;7:235. doi: 10.4236/ojo.2017.79025.

16. Cherrad T, Louaste J, Kasmaoui el H, Bousbaa H, Rachid K. Neglected bilateral rupture of the patellar tendon: A case report. $J$ Clin Orthop Trauma. 2015;6(4):296-9. doi: 10.1016/j.jcot.2015.04.003. [PubMed: 26566349]. [PubMed Central: PMC4600838]. 